\title{
Tight Junction Protein 1 Dysfunction Contributes to Cell Motility in Bladder Cancer
}

\author{
KUO-WANG TSAI ${ }^{1,2,3}$, WEI-TING KUO ${ }^{4,5,6}$ and SHAW-YEU JENG ${ }^{4,5}$ \\ ${ }^{1}$ Department of Medical Education and Research, \\ Kaohsiung Veterans General Hospital, Kaohsiung, Taiwan, R.O.C.; \\ ${ }^{2}$ Department of Chemical Biology, National Pingtung University of Education, Pingtung, Taiwan, R.O.C.; \\ ${ }^{3}$ Institute of Biomedical Sciences, National Sun Yat-Sen University, Kaohsiung, Taiwan, R.O.C.; \\ ${ }^{4}$ Division of Urology, Department of Surgery, Kaohsiung Veterans General Hospital, Kaohsiung, Taiwan, R.O.C.; \\ ${ }^{5}$ Division of Urology, Department of Surgery, Tri-Service General Hospital, \\ National Defense Medical Center, Taipei, Taiwan, R.O.C.; \\ ${ }^{6}$ School of Medicine, National Yang-Ming University, Taipei, Taiwan, R.O.C.
}

\begin{abstract}
Background/Aim: Bladder cancer is the most common malignancy involving the urinary system. The mortality rate in late stages remains high, thus the development of effective biomarkers for diagnosis or prognosis is required in order to improve patient survival rates. Tight junction protein 1 (TJP1) is a membraneassociated protein that helps modulate cell-cell contact. However, the role of TJP1 in bladder cancer progression remains unclear. Materials and Methods: The expression levels of TJP1 and miR-455-5p were examined by analyzing The Cancer Genome Atlas database. The biological role of TJP1 and miR-455-5p were assessed in T24 cells with siTJP1 or miR-455-5p mimics transfection, respectively. Results: High levels of expression of TJP1 were significantly correlated with poor lymph node metastasis ( $p N$ stage; $p=0.004)$. Knockdown of the TJP1 gene expression led to significant decrease of the growth and invasion of T24 cells. Using a bioinformatics approach, miR-455-5p was shown to suppress TJP1 expression by directly targeting its 3' prime untranslated region in bladder cancer cells. The ectopic expression of miR-455-5p revealed that bladder cancer cell migration, invasion, and proliferation were significantly suppressed. Conclusion: In summary, our results indicate
\end{abstract}

Correspondence to: Kuo-Wang Tsai, Department of Medical Education and Research, Kaohsiung Veterans General Hospital, Kaohsiung 813, Taiwan, R.O.C. Tel: +886 73422121, Fax: +886 73468056, e-mail: kwtsai6733@gmail.com orkwtsai@ksvgh.gov.tw and Shaw-Yeu Jeng, Department of Surgery, Kaohsiung Veterans General Hospital, Kaohsiung 813, Taiwan, R.O.C. Tel: +886 73422121, Fax: +886 73468056, e-mail: syjeng@ vghks.gov.tw

Key Words: Bladder cancer, TJP1, miR-455. that dysfunction of the miR-455-TJP1 axis is involved in bladder cancer cell growth and metastasis. These findings highlight potential therapeutic targets or putative biomarkers for bladder cancer.

Bladder cancer is the most common malignancy involving the urinary system. Urothelial carcinoma is the predominant histological type, accounting for approximately $90 \%$ of all bladder cancers. Less commonly, urothelial cancers can arise in the renal pelvis, ureter, or urethra. Bladder cancer is more common in men than in women with a ratio of approximately $3: 1$, and it is the fourth most common solid malignancy in men. An estimated 16,000 deaths resulted from bladder cancer in 2015, predominantly men $(1,2)$. Bladder cancer primarily affects Caucasian Americans and those aged $>65$ years, with relatively stable mortality rates since 1975 (2). No currently accepted genetic or inheritable cause of bladder cancer is known; however, genomic instability and genetic pathway mutations and alterations may be involved in bladder carcinogenesis $(3,4)$.

Tight junction protein 1 (TJP1) is a component of tight junctions that can regulate actin cytoskeleton remodeling (5). TJP1 expression is aberrant during tumor progression and plays a crucial role in modulating cancer migration and invasion (6-11). The expression levels of TJP1 decreased in cancer tissues compared with adjacent normal tissues, and TJP1 expression plays a suppressive role in modulating cancer cell growth or invasion, in breast cancer, colorectal carcinoma, lung cancer, and hepatocellular carcinoma cells $(6,9,10,12-14)$. However, few studies have reported that TJP1 might promote cancer cell motility in melanoma, gastric cancer, and pancreatic cancer cells $(7,8,15)$.

MicroRNAs (miRNAs) are small RNA molecules that perform vital regulatory functions in several physiological 
activities (16). In bladder cancer, numerous dysfunctional miRNAs have been reported to play tumor-suppressive and oncogenic roles in regulating cancer cell proliferation and invasion, including miR-101, miR-149-3p, miR-154, and miR429 (17-20). A study reported that miR-144 could silence TJP1 expression by directly targeting its three prime untranslated (3'UTR) region (21). However, the detailed role of TJP1 in bladder cancer progression remains unknown. In the present study, the biological role of TJP1 expression in bladder cancer was assessed. Our findings provide a therapeutic target or putative biomarker for future bladder cancer treatments.

\section{Materials and Methods}

Cell line. Two human bladder cancer cell lines, T24 and RT4, were obtained from the American Type Culture Collection and maintained in Dulbecco's modified Eagle's medium supplemented with $10 \%$ inactivated fetal bovine serum (Invitrogen, Carlsbad, CA, USA). Total RNA was extracted using TRIzol reagent (Invitrogen), according to the instruction manual. Cells were briefly homogenized in $1 \mathrm{ml}$ of TRIzol reagent and mixed with $0.2 \mathrm{ml}$ of chloroform to extract protein. RNA was precipitated using $0.5 \mathrm{ml}$ of isopropanol. The concentration, purity, and amount of total RNA were determined using a Nanodrop 1000 spectrophotometer (Nanodrop Technologies Inc., Wilmington, DE, USA).

Real-time reverse transcription polymerase chain reaction. A total of $2 \mu \mathrm{g}$ of total RNA (DNase I treatment) was reverse transcribed using oligo (dT)15 primers and SuperScript III Reverse Transcriptase according to the instruction manual of the manufacturer (Invitrogen). The reaction was performed by first incubating the enzyme at $42^{\circ} \mathrm{C}$ for $1 \mathrm{~h}$. Then, the enzyme was inactivated through incubation at $85^{\circ} \mathrm{C}$ for $5 \mathrm{~min}$. Complementary DNA was used for real-time reverse transcription (RT) polymerase chain reaction (RT-PCR) analysis with gene-specific primers, and gene expression was detected using a SYBR Green I assay (Applied Biosystems, Foster City, CA, USA). The sequence of primers was as follows: TJP1-F: 5'-ACCAGTAAGTCGTCCTGATCC-3'; TJP1R: 5'-TCGGCCAAATCTTCTCACTCC-3'; GAPDH-F: 5'-TGCAC CACCAACTGCTTAGC-3'; GAPDH-R: 5'-GGCATGGACTGTGG TCATGAG-3'.

Stem-loop RT-PCR. Next, $1 \mu \mathrm{g}$ of total RNA was reverse transcribed by performing a stem-loop RT reaction by using microRNA RT primers and SuperScript III Reverse Transcriptase according to the instruction manual (Invitrogen). The reaction was performed using the following incubation conditions: $16^{\circ} \mathrm{C}$ for $30 \mathrm{~min}$, followed by 50 cycles of $20^{\circ} \mathrm{C}$ for $30 \mathrm{sec}, 42^{\circ} \mathrm{C}$ for $30 \mathrm{sec}$, and $50^{\circ} \mathrm{C}$ for $1 \mathrm{sec}$. The enzyme was subsequently inactivated through incubation at $85^{\circ} \mathrm{C}$ for 5 min. Gene expression was detected using an SYBR Green I assay (Applied Biosystems), and the expression level of miR-455-5p was normalized to that of $\mathrm{U} 6(\Delta \mathrm{Ct}=\mathrm{miR}-455-5 \mathrm{p} \mathrm{Ct}-\mathrm{U} 6 \mathrm{Ct})$. To simplify data presentation, relative expression values were multiplied by $10^{5}$ miR-455-5p-RT: 5'-CTCAACTGGTGTCGTGGAGTCGGCAATT CAGTTGAGCGATGTAG-3'; miR-455-5p-GSF: 5'-CGGCGGTATG TGCCTTTGGAGT-3'; Universal reverse: 5'-CTGGTGTCGT GGAGTCGGCAATTC-3'; U6-F: 5'-CTCGCTTCGGCAGCACA-3'; U6-R: 5'-AACGCTTCACGAATTTGCGT-3.
Small interfering RNA transfection. Small interfering RNA (siRNA) oligonucleotides targeting TJP1 (siTJP1, sense: 5'-CCAGUAUCUG AUAAUGAAGTT-3' and antisense: 5'-AUUCAUUAUCAGAU ACUGGTT-3') and a scrambled oligo as a negative control were designed and synthesized by GenDiscovery Biotechnology (Taipei, Taiwan). The cells were transfected with a final concentration (10 mM) of individual siRNA or control by using Lipofectamine RNAiMAX (Invitrogen; Thermo Fisher Scientific Inc., Waltham, MA, USA). Twenty-four hours after transfection h, RNA was extracted and knockdown efficiency was evaluated by real-time RT-PCR.

Ectopic expression of miR-455-5p through transfection with mimics. The bladder cancer cells were seeded in a 25-T flask at a density of $1 \times 10^{6}$ cells $/ \mathrm{ml}$, and these cells were transfected with $10 \mathrm{nM}$ of miRNA-455-5p mimics (5'- UAUGUGCCUUUGGACUACAUCG $-3^{\prime}$ ) or an appropriate miRNA mimic control (GenDiscovery Biotechnology Inc., Taipei, Taiwan) by using Lipofectamine RNAiMAX reagent (Invitrogen). After $24 \mathrm{~h}$, the transfected cells were harvested, and expression levels were examined through stemloop RT-PCR.

Western blotting. The cells were washed with phosphate-buffered saline (PBS) and then lysed with a lysis buffer $(50 \mathrm{mM}$ Tris- $\mathrm{HCl}$ at $\mathrm{pH} 8.0,150 \mathrm{mM} \mathrm{NaCl}, 1 \% \mathrm{NP}-40,0.02 \%$ sodium azide, $1 \mu \mathrm{g} / \mathrm{ml}$ of aprotinin, and $1 \mathrm{mM} \mathrm{PMSF}$ ) at $4^{\circ} \mathrm{C}$ for $30 \mathrm{~min}$. The lysate was then collected and centrifuged to remove cell debris. Protein assays were performed using a Bio-Rad Protein Assay kit (Bio-Rad Inc., Hercules, CA, USA) based on the Bradford dye-binding procedure. Protein samples $(60 \mu \mathrm{g})$ were separated through sodium dodecyl sulfate-polyacrylamide gel electrophoresis in $10 \%$ resolving gel by using a Mini-PROTEAN 3 cell apparatus. The proteins were then electrotransferred onto nitrocellulose membranes (NEF1002001PK, PerkinElmer, Inc., Waltham, MA, USA). After blocking at $4^{\circ} \mathrm{C}$ overnight by using PBS-Tween containing 5\% skim milk, the membranes were incubated with anti-TJP1 (1:1,000, D7D12, Cell Signaling Technology, MA, USA) or ACTB (1:2,000, MAB1501, EMD Millipore, Billerica, MA, USA) for $1 \mathrm{~h}$ in PBS-Tween containing 5\% skim milk. The membranes were then incubated with anti- rabbit or mouse $\operatorname{IgG}$ horseradish peroxidase-conjugated secondary antibodies (1:10,000, Roche Molecular Biochemicals, Indianapolis, IN, USA) for $1 \mathrm{~h}$ at room temperature. After being washed three times with PBS-Tween, immunoreactive bands were detected using an enhanced chemiluminescence kit (Advansta, Menlo Park, CA, USA).

Cell proliferation and colony formation assay. For cell proliferation analysis, 1000 living cells were plated onto 96-well plates and transfected with miR-455-5p mimics, siTJP1, or a control. Cell growth was determined at $0,1,2,3$, and 4 days by using a CellTiter-Glo One Solution Assay (Promega Corporation, Madison, WI, USA). In the clonogenic assay, 8,000 bladder cancer cells were seeded in a 6-well plate and transfected with siTJP1, miRNA-455$5 \mathrm{p}$ mimics, or a control. The cells were incubated in a $\mathrm{CO}_{2}$ incubator at $37^{\circ} \mathrm{C}$ for 2 weeks until substantially sized colonies were formed. The medium was removed, cells were fixed in $1 \mathrm{ml}$ of $10 \%$ formaldehyde solution, and the plates were incubated at room temperature for $2 \mathrm{~min}$. After removal of the fixation solution, $1 \mathrm{ml}$ of crystal violet solution was added, and the plates were incubated at room temperature for $2 \mathrm{~h}$. Subsequently, crystal violet solution was removed, and the plates were rinsed. The plates were air-dried 
at room temperature, and $1 \mathrm{ml}$ of $10 \%$ acetic acid was added to each well to dissolve crystal violet. The absorbance of individual wells was determined at $595 \mathrm{~nm}$ by using a Multiskan FC (Thermo Scientific).

Migration and invasion assay. The cells were tested for migration and invasion abilities in vitro in transwell chambers (Costar, Lowell, MA, USA). The lower side or the upper side of the polycarbonate membranes (containing 8- $\mu \mathrm{m}$ pores) of the transwell were coated with $50 \mu \mathrm{g} / \mathrm{ml}$ of type I collagen or $80 \mu \mathrm{g}$ per well of Matrigel and then used for migration or invasion assays, respectively. T24 cells were transfected with siTJP1, miR-455-5p mimics, or a control. Subsequently, the cells were added to the upper chamber of a transwell. After incubation for $24 \mathrm{~h}$ at $37^{\circ} \mathrm{C}$, the cells at the lower side were prepared for Giemsa staining. The level of migration or invasion was determined using a microscope at $200 \times$ magnification.

miRNA target candidates and luciferase reporter assay. The predicted targets of miRNAs were determined using the TargetScan prediction tool (36). In this study, we identified 25 candidate miRNAs that might target the 3'-UTR of TJP1. The 3'-UTR sequences and seed region mutant of TJP1 were cloned into a pMIR-REPROT vector (AM5795, Thermo Scientific). Subsequently, the pMIR-REPROT-TJP1 or pMIR-REPROT$\mathrm{TJP} 1_{\text {(mutant) }}$ vector was cotransfected with or without the miR-455$5 p$ expression vector into a $293 \mathrm{~T}$ cell line by using Lipofectamine 2000 (Invitrogen). After 24-h transfection, cell lysates were used to measure luciferase activity by using a Dual-Glo Luciferase Reporter Assay System (Promega).

\section{Results}

TJPl was highly expressed in invasive bladder cancer cell lines. In this study, RNA transcriptome data from 379 cases of bladder cancer were downloaded from The Cancer Genome Atlas database. Using these data, we analyzed the correlation between clinicopathological features and TJP1 expression. As listed in Table I, a high TJP1 expression level was significantly associated with poor lymph node metastasis ( $\mathrm{pN}$ stage, $p=0.004)$. As illustrated in Figure 1A, the expression level of TJP1 was significantly higher in bladder cancer patients with lymph node metastasis than in bladder cancer patients without lymph node metastasis (N0 vs. N2 + N3, $p=0.008$ ). Furthermore, the expression level of TJP1 in two bladder cancer cell lines, namely T24 and RT4 was examined. As illustrated in Figure $1 \mathrm{~B}$ and $1 \mathrm{C}$, both the mRNA and protein expression levels of TJP1 in T24 cells were higher than those in RT4 cells. To determine whether TJP1 expression is associated with the invasive ability of bladder cancer cells, the invasion ability of T24 and RT4 cells was examined by performing an invasion assay. As illustrated in Figure 1D-F, T24 cells were more invasive compared with RT4 cells, implying that TJP1 might contribute to bladder cancer migration and invasion. These results indicate that TJP1 might play a crucial role in modulating bladder cancer motility.
Table I. Correlation of TJP1 expression with the clinicopathological characteristics of 379 cases of bladder cancer

\begin{tabular}{lcccc}
\hline Variables & \multicolumn{4}{c}{ TJP1 (n=379) } \\
\cline { 2 - 5 } & No. (\%) & Mean \pm SD & Median & $p$-Value \\
\hline Pathology stage & & & & \\
I + II & $115(30.3)$ & $1752.35 \pm 876.70$ & 1626.74 & $0.627^{\mathrm{a}}$ \\
III + IV & $264(69.7)$ & $1800.80 \pm 896.29$ & 1677.59 & \\
pT stage (n=377) & & & & \\
T1 + T2 & $131(34.7)$ & $1744.79 \pm 861.77$ & 1612.00 & $0.527^{\mathrm{a}}$ \\
T3 + T4 & $246(65.3)$ & $1805.84 \pm 908.15$ & 1689.39 & \\
pN stage (n=378) & & & & \\
N0 + N1 & $297(78.6)$ & $1701.78 \pm 755.00$ & 1631.13 & $0.004^{\mathrm{b}}$ \\
N2 + N3 & $81(21.4)$ & $2100.77 \pm 1224.88$ & 1784.57 & \\
pM stage (n=305) & & & & \\
M0 & $295(96.7)$ & $1768.98 \pm 777.00$ & 1692.65 & $0.256^{\mathrm{a}}$ \\
M1 & $10(3.3)$ & $2055.12 \pm 949.92$ & 2028.23 & \\
Gender & & & & \\
Male & $279(73.6)$ & $1793.34 \pm 922.35$ & 1673.53 & $0.792^{\mathrm{a}}$ \\
Female & $100(26.4)$ & $1765.92 \pm 794.73$ & 1638.11 & \\
\hline
\end{tabular}

${ }^{\mathrm{a}} p$-value were estimated by the Student's $t$-test. ${ }^{\mathrm{b}} p$-value were estimated by Mann-Whitney $U$-test.

TJP1 expression in bladder cancer growth and invasion. To examine the role of TJP1 in bladder cancer, TJP1 was knocked down in T24 cells by using a siTJP1 approach; proliferation, colony formation, and migration were assessed using an invasion assay. As shown in Figure 2A, expression of TJP1 clearly decreased after siTJP1 transfection for $48 \mathrm{~h}$. Furthermore, the effects of TJP1 knockdown on T24 cell growth, including proliferation and colony formation were examined. Our data revealed that knockdown of TJP1 expression significantly suppressed colony formation and proliferation of T24 cells (Figure 2B-D). Transwell assay findings indicated that TJP1 knockdown could significantly suppress migration and invasion of T24 cells (Figure 2E-G).

MiR-455 silenced TJP1 expression by targeting 3'-UTR. The aforementioned results suggested that TJP1 expression contributes to bladder cancer growth and cell motility. Furthermore, high TJP1 expression was correlated with unfavorable bladder cancer prognosis. However, the mechanism underlying TJP1 dysregulation remains unclear. Therefore, putative miRNAs that could target the 3'-UTR of TJP1 were identified by using a bioinformatics approach. A total of 37 putative miRNAs were identified using TargetScan prediction tools. The expression levels of real target genes were negatively correlated with TJP1 expression in bladder cancer. Among them, the expression level of miR-455-5p was negatively correlated with that of TJP1 in bladder cancer $(\mathrm{r}=-0.12)$. In addition, the expression of miR-455-5p was significantly lower in bladder cancer with lymph node 
(A)

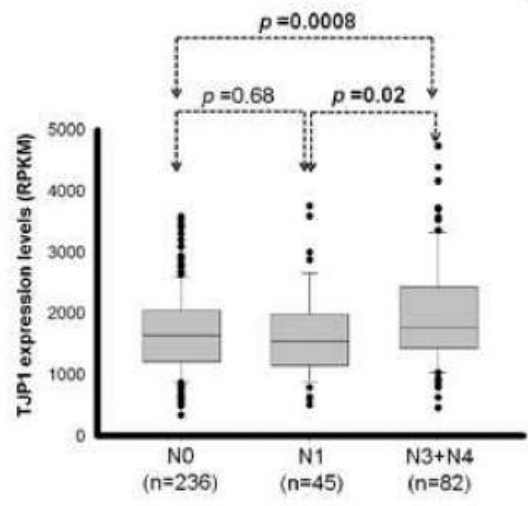

(D)

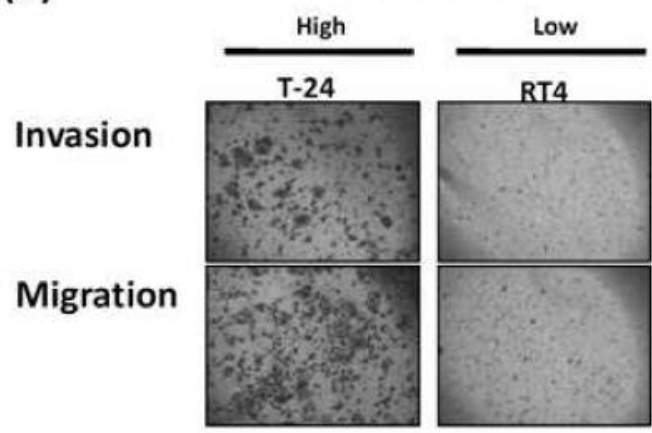

(B)

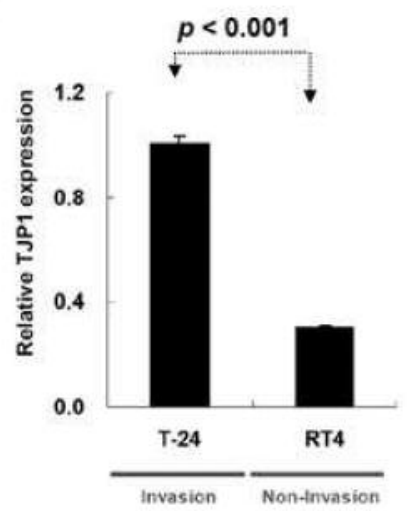

(E)

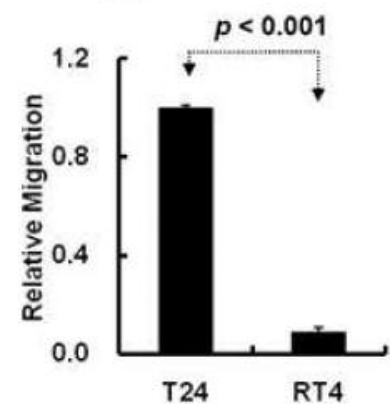

(C)

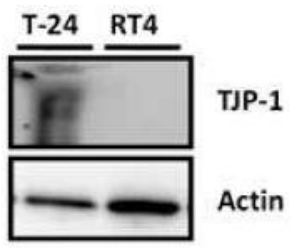

(F)

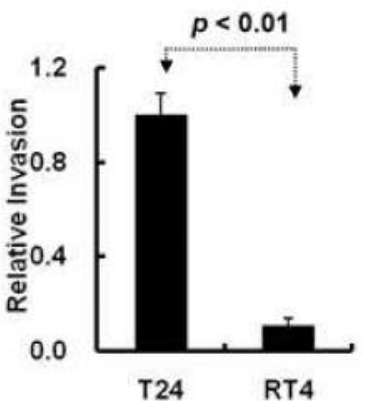

Figure 1. TJP1 expression is correlated with bladder cancer lymph node metastasis. (A): The expression level of TJP1 was compared between bladder cancer patients with lymph node metastasis and those without lymph node metastasis by using the TCGA database. (B) and (C): The expression level of TJP1 was examined in T24 and RT4 cells by real-time RT-PCR and western blotting. (D), (E), and (F): The migration/invasion ability of T24 and RT4 cells was examined using a transwell assay. Cell images of a representative experiment are shown, and graph values were quantified using Ascent software. Data are reported as the number of colonies relative to the control (mean $\pm S D$ ).

metastasis ( $\mathrm{N} 0$ vs. $\mathrm{N} 2+\mathrm{N} 3, p=0.001$, Figure $3 \mathrm{~A})$. To determine whether miR-455-5p silences TJP1 expression, the expression level of TJP1 was examined in T24 cells after miR-455-5p transfection. As shown in Figure 2B and 2C, miR-455-5p could suppress TJP1 protein expression. The results of the luciferase reporter assay revealed that miR-455$5 \mathrm{p}$ suppressed luciferase activity by directly targeting the 3'UTR of TJP1 (Figure 3D and E). When the binding sites of miR-455-5p were mutated, the luciferase activity was restored (Figure 3F). Further assessment of the biological function of miR-455-5p revealed that its ectopic expression could suppress bladder cancer cell proliferation and colony formation (Figure 4A-C). Furthermore, miR-455-5p was found to inhibit migration and invasion of T24 cells (Figure 4D-F). These results were consistent with the finding that growth and invasion abilities were significantly inhibited in T24 cells after TJP1 knockdown. Our study is the first to report that miR-455-5p can inhibit bladder cancer cell growth and invasion by silencing TJP1 expression.

\section{Discussion}

Studies have reported frequent down-regulation of TJP1 in cancer tissues compared with adjacent normal tissues, including in breast cancer, colorectal carcinoma, lung cancer, and hepatocellular carcinoma $(6,9,10,12-14)$. Furthermore, TJP1 protein is generally considered to be a tumor suppressor, and high TJP1 expression is associated with a favorable prognosis $(6,9,10,12-14)$. However, some studies have reported contradictory results indicating that TJP1 might play an oncogenic role in cancer progression, such as in melanoma gastric cancer and pancreatic cancer cells $(7,8,15)$. This study, indicates that high TJP1 expression correlates with lymph node metastasis. Moreover, TJP1 knockdown significantly suppressed bladder cancer cell growth and migration, suggesting that TJP1 plays an oncogenic role in the growth of bladder cancer cells. Similarly, Zhang et al. reported that TJP1 contributes to ovarian follicular development and promotes ovarian cancer growth (22). Transforming growth factor-beta 
(A)

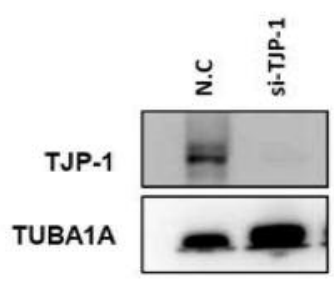

(C)

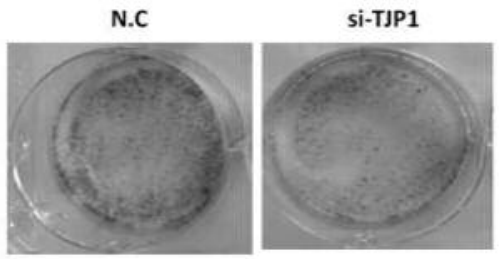

(E)

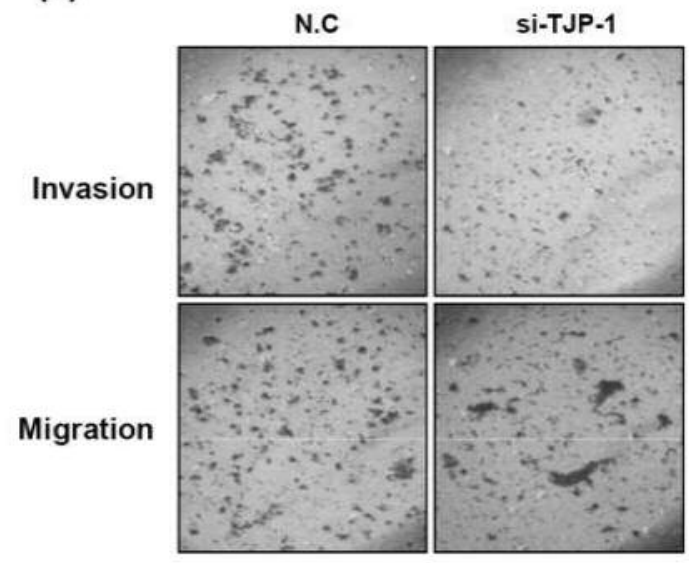

(B)

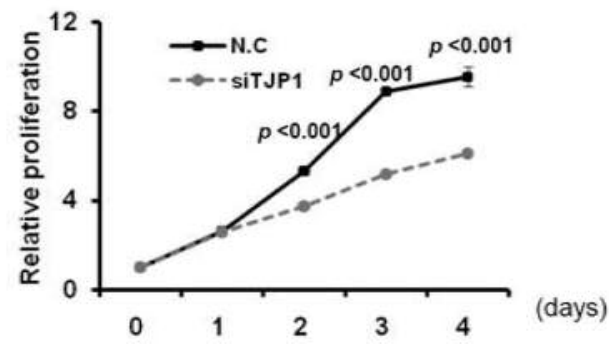

(D)

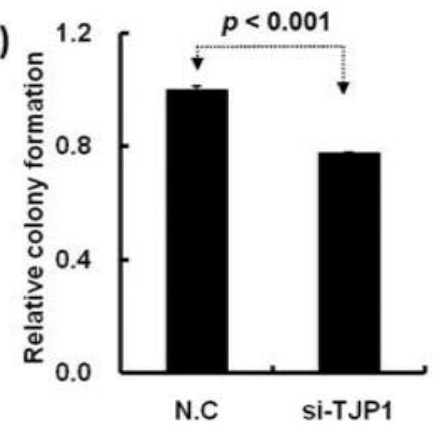

(F)
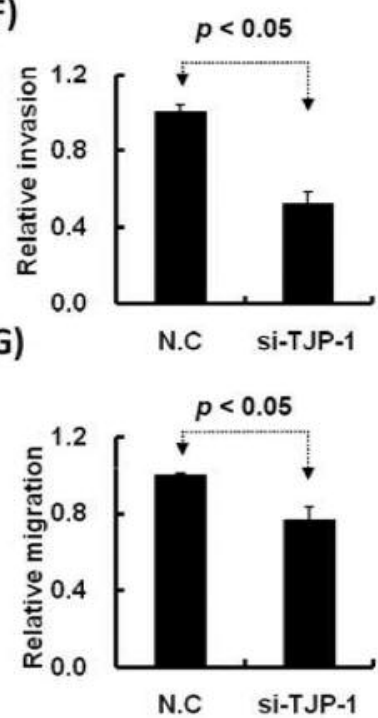

Figure 2. Biological function of TJP1 knockdown is assessed in T24 cells. (A): The expression level of TJP1 was examined in T24 cells transfected with siTJP1 or a control by western blotting. (B): After TJP1 knockdown, cell proliferation was measured at various points $(0,1,2,3$, and 4 days) and was compared with the scramble control. $(C)$ and $(D)$ : Colony formation assay was performed in T24 cells after transfection with siTJP1 or a control for 2 weeks. The cells were fixed and stained with crystal violet solution, and the relative colony formation was quantified. (E): After transfecting with siTJP1 or a control, the migration and invasion ability of the cells was examined using a transwell assay. The invading and migrating cells were stained with crystal violet solution and enumerated by counting three fields under a phase-contrast microscope. $(F)$ and $(G)$ : The relative migration and invasion ability were quantified in T24 cells with and without TJP1 knockdown.

(TGF- $\beta$ ) is a driving force behind the epithelial-mesenchymal transition in human cancer cells (23). Lee et al. noted that TJP1 expression significantly increased the progression of TGF- $\beta$ induced A549 epithelial-mesenchymal transition (24). The aforementioned results suggest that TJP1 can be involved in the promotion cancer cell metastasis in lung cancer cells. TJP1 protein can exist in the cell membrane, cytoplasm, or nucleus of human cancer cells, playing specific roles in these different subcellular compartments $(14,25,26)$. Polette et al. reported that TJP1 protein is localized in different cellular systems and 
(A)

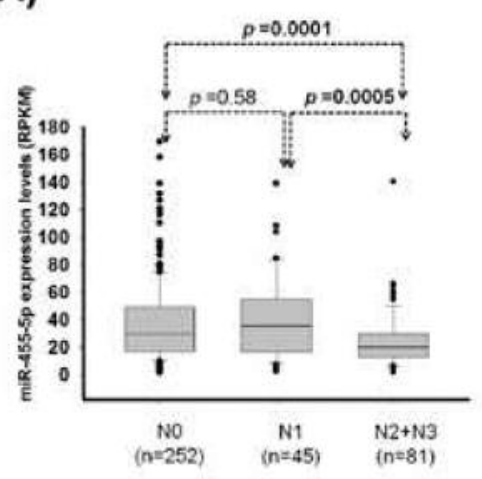

(B)

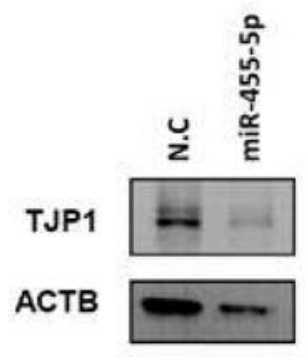

(C)

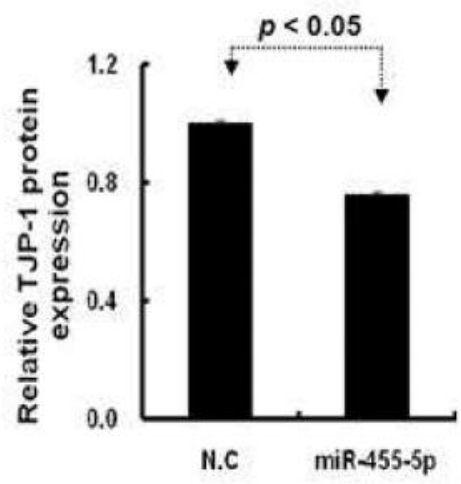

(D)

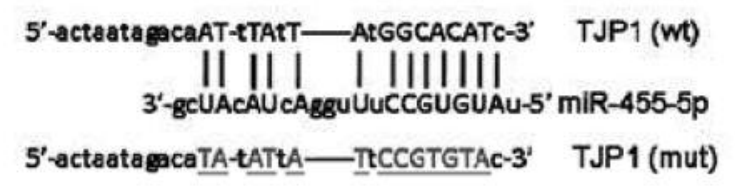

(E)

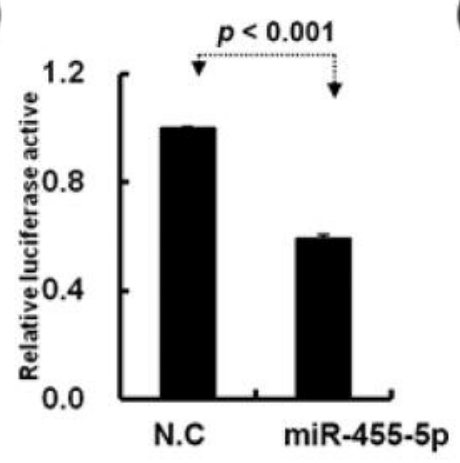

(F)

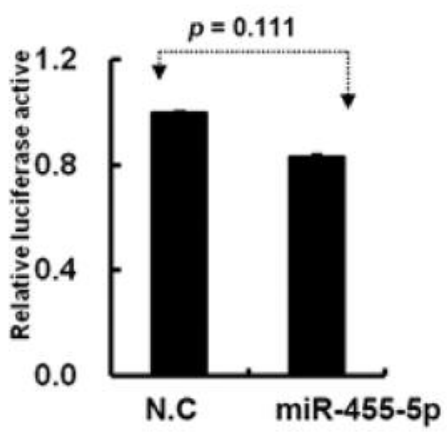

Figure 3. miR-455-5p targeting 3'-UTR of TJP1 is identified through bioinformatics and experimental approaches. (A): The expression level of miR455-5p was examined in bladder cancer with and without lymph node metastasis by analyzing the TCGA database. (B) and $(C)$ : The expression level of TJP1 was examined in T24 cells by using miR-455-5p mimics or control transfection by western blotting. (D): The miR-455-5p target

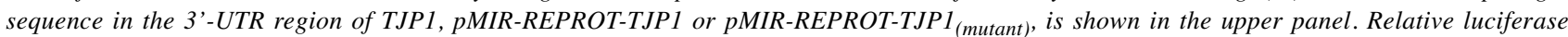
activity of the reporter with the 3'-UTR $(E)$ or seed region mutant of TJP1 $(F)$ was determined after cotransfection of $293 T$ cancer cells with miR455-5p or a control. Firefly luciferase activity served as a normalization control.

that the delocalization of TJP1 protein in cytoplasmic or nuclear compartments confers promigratory or invasive and protumoral roles on TJP1 (14). In melanoma and breast cancer cells, TJP1 has been demonstrated to have an oncogenic role by promoting tumor invasion $(8,27)$. Lesage et al. reported that TJP1 protein significantly shifted from the membrane to cytoplasm in A549 cells following treatment with TGF- $\beta$ (28). Furthermore, TJP1 can accelerate tumor angiogenesis by regulating the NFkB-CXCL8 axis (28). Taken together, these findings indicate that TJP1 plays a distinct role in human cancer cells, and its subcellular distribution contributes to its functions. Cytoplasmic TJP1 might possess an oncogenic function in regulating cancer cell motility. In bladder cancer cells, TJP1 protein is located in the cell membrane and cytoplasm (data not shown); however, the detailed mechanism underlying the involvement of cytoplasmic TJP1 in bladder cancer cell metastasis remains unclear.

miR-455-5p plays a tumor-suppressive role in cancer cell growth and migration through silencing RAB18, EGFR, and CDK14 (29-31). By contrast, miR-455-5p plays an oncogenic role by promoting cancer cell growth and migration in melanoma cancer, lung cancer, colon cancer, and oral cancer by targeting CPEB1, SOCO3, galectin-9, and UBE2B (32-35). Taken together, these studies suggest that miR-455-5p might have a dual function depending on the target. In this study, we demonstrated for the first time that miR-455-5p can suppress TJP1 expression by directly targeting its 3'-UTR. Furthermore, miR-455-5p expression 
(B)

(A)

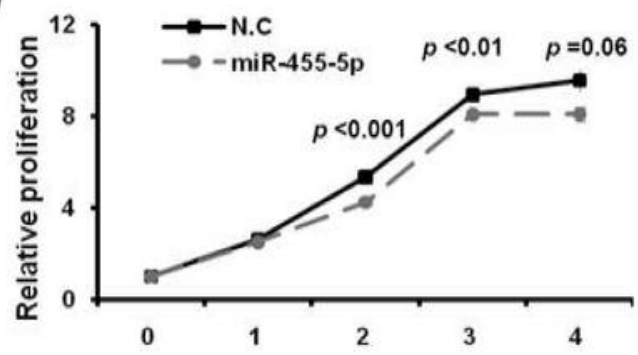

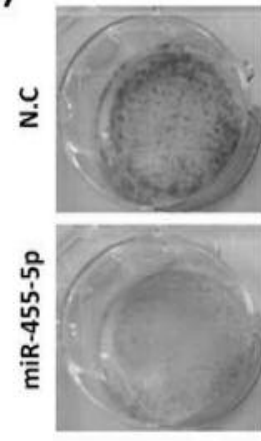

(C)

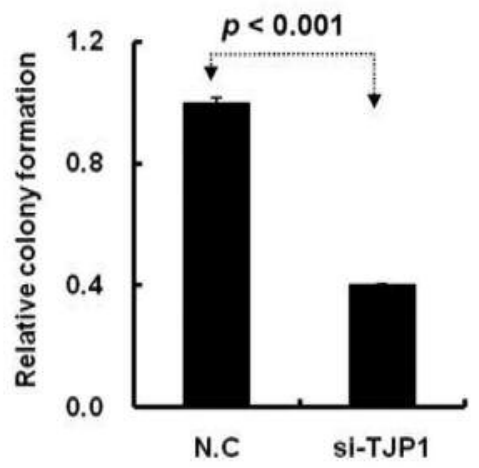

(D)

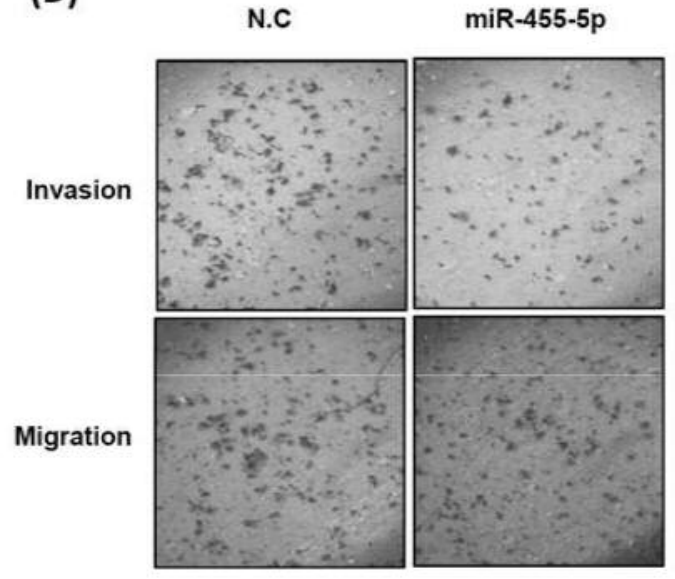

(E)

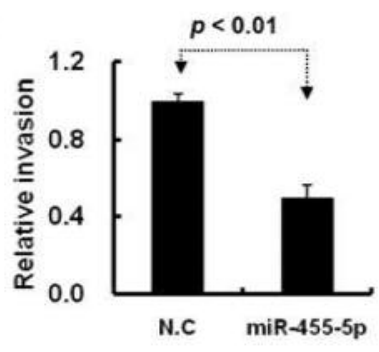

(F)

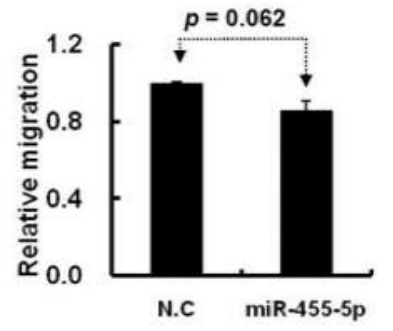

Figure 4. Biological function of miR-455-5p expression is assessed in T24 cells. (A): After ectopic miR-455-5p expression, cell proliferation was measured at various points $(0,1,2,3$, and 4 days) and compared with the scramble control. $(B)$ and $(C)$ : Colony formation assay was performed in T24 cells after transfection with miR-455-5p mimics or a control for 2 weeks. The cells were fixed and stained with crystal violet solution, and the relative colony formation was quantified. (D): After transfecting with miR-455-5p mimics or a control, the migration and invasion abilities of cells were examined using a transwell assay. The invading and migrating cells were stained with crystal violet solution and were enumerated by counting three fields under a phase-contrast microscope. $(E)$ and $(F)$ : The relative migration and invasion abilities were quantified in T24 cells with and without miR-455-5p overexpression.

can suppress bladder cancer cell motility and growth. Therefore, the miR-455-5p-TJP1 axis may involve regulating bladder cancer cell growth and migration.

In summary, it is reported for the first time that miR-455TJP1 axis dysfunction is involved in bladder cancer cell metastasis and growth. These findings serve to highlight potential therapeutic targets or putative biomarkers for bladder cancer.

\section{Conflicts of Interest}

The Authors declare that they have no competing interests.

\section{Acknowledgements}

This work was supported by grants from Kaohsiung Veterans General Hospital, Taiwan (VGHKS-106-087, VGHKS107-104, and VGHKS107-017).

\section{References}

1 Siegel RL, Miller KD and Jemal A: Cancer statistics, 2015. CA Cancer J Clin 65: 5-29, 2015.

2 Abdollah F, Gandaglia G, Thuret R, Schmitges J, Tian Z, Jeldres C, Passoni NM, Briganti A, Shariat SF, Perrotte P, Montorsi F, Karakiewicz PI and Sun M: Incidence, survival and mortality 
rates of stage-specific bladder cancer in United States: a trend analysis. Cancer Epidemiol 37: 219-225, 2013.

3 Garcia-Closas M, Malats N, Silverman D, Dosemeci M, Kogevinas M, Hein DW, Tardon A, Serra C, Carrato A, GarciaClosas R, Lloreta J, Castano-Vinyals G, Yeager M, Welch R, Chanock S, Chatterjee N, Wacholder S, Samanic C, Tora M, Fernandez F, Real FX and Rothman N: NAT2 slow acetylation, GSTM1 null genotype, and risk of bladder cancer: results from the Spanish Bladder Cancer Study and meta-analyses. Lancet 366: 649-659, 2005.

4 Ploussard G, Dubosq F, Soliman H, Verine J, Desgrandchamps F, De The H and Mongiat-Artus P: Prognostic value of loss of heterozygosity at chromosome $9 \mathrm{p}$ in non-muscle-invasive bladder cancer. Urology 76: 513 e513-518, 2010.

5 Itoh M, Nagafuchi A, Moroi S and Tsukita S: Involvement of ZO-1 in cadherin-based cell adhesion through its direct binding to alpha catenin and actin filaments. J Cell Biol 138: 181-192, 1997.

6 Hoover KB, Liao SY and Bryant PJ: Loss of the tight junction MAGUK ZO-1 in breast cancer: relationship to glandular differentiation and loss of heterozygosity. Am J Pathol 153: 1767-1773, 1998.

7 Kleeff J, Shi X, Bode HP, Hoover K, Shrikhande S, Bryant PJ, Korc M, Buchler MW and Friess H: Altered expression and localization of the tight junction protein $\mathrm{ZO}-1$ in primary and metastatic pancreatic cancer. Pancreas 23: 259-265, 2001.

8 Smalley KS, Brafford P, Haass NK, Brandner JM, Brown E and Herlyn M: Up-regulated expression of zonula occludens protein1 in human melanoma associates with $\mathrm{N}$-cadherin and contributes to invasion and adhesion. Am J Pathol 166: 15411554, 2005.

9 Ni S, Xu L, Huang J, Feng J, Zhu H, Wang G and Wang X: Increased ZO-1 expression predicts valuable prognosis in nonsmall cell lung cancer. Int J Clin Exp Pathol 6: 2887-2895, 2013.

10 Nagai T, Arao T, Nishio K, Matsumoto K, Hagiwara S, Sakurai T, Minami Y, Ida H, Ueshima K, Nishida N, Sakai K, Saijo N, Kudo K, Kaneda H, Tamura D, Aomatsu K, Kimura H, Fujita Y, Haji S and Kudo M: Impact of Tight Junction Protein ZO-1 and TWIST Expression on postoperative survival of patients with hepatocellular carcinoma. Dig Dis 34: 702-707, 2016.

11 Hsu YL, Hung JY, Chang WA, Lin YS, Pan YC, Tsai PH, Wu $\mathrm{CY}$ and Kuo PL: Hypoxic lung cancer-secreted exosomal miR23a increased angiogenesis and vascular permeability by targeting prolyl hydroxylase and tight junction protein ZO-1. Oncogene 36: 4929-4942, 2017.

12 Kaihara T, Kusaka T, Nishi M, Kawamata H, Imura J, Kitajima K, Itoh-Minami R, Aoyama N, Kasuga M, Oda Y, Hattori M and Fujimori T: Dedifferentiation and decreased expression of adhesion molecules, E-cadherin and ZO-1, in colorectal cancer are closely related to liver metastasis. J Exp Clin Cancer Res 22: 117-123, 2003.

13 Kimura Y, Shiozaki H, Hirao M, Maeno Y, Doki Y, Inoue M, Monden T, Ando-Akatsuka Y, Furuse M, Tsukita S and Monden M: Expression of occludin, tight-junction-associated protein, in human digestive tract. Am J Pathol 151: 45-54, 1997.

14 Polette M, Mestdagt M, Bindels S, Nawrocki-Raby B, Hunziker W, Foidart JM, Birembaut P and Gilles C: Beta-catenin and ZO1: shuttle molecules involved in tumor invasion-associated epithelial-mesenchymal transition processes. Cells Tissues Organs 185: 61-65, 2007.
15 Resnick MB, Gavilanez M, Newton E, Konkin T, Bhattacharya B, Britt DE, Sabo E and Moss SF: Claudin expression in gastric adenocarcinomas: a tissue microarray study with prognostic correlation. Hum Pathol 36: 886-892, 2005.

16 Yekta S, Shih IH and Bartel DP: MicroRNA-directed cleavage of HOXB8 mRNA. Science 304: 594-596, 2004.

17 Zhao X, Ji Z, Xie Y, Liu G and Li H: MicroRNA-154 as a prognostic factor in bladder cancer inhibits cellular malignancy by targeting RSF1 and RUNX2. Oncol Rep 38: 2727-2734, 2017.

18 Yang J, Liu Y, He A, Liu Y, Wu J, Liao X, Lv Z, Wang F and Mei H: Hsa-miR-429 promotes bladder cancer cell proliferation via inhibiting CDKN2B. Oncotarget 8: 68721-68729, 2017.

19 Yang D, Du G, Xu A, Xi X and Li D: Expression of miR-149$3 p$ inhibits proliferation, migration, and invasion of bladder cancer by targeting S100A4. Am J Cancer Res 7: 2209-2219, 2017.

20 Li B, Xie F, Zheng FX, Jiang GS, Zeng FQ and Xiao XY: Overexpression of CircRNA BCRC4 regulates cell apoptosis and MicroRNA-101/EZH2 signaling in bladder cancer. J Huazhong Univ Sci Technolog Med Sci 37: 886-890, 2017.

21 Hou Q, Huang Y, Zhu S, Li P, Chen X, Hou Z and Liu F: MiR144 Increases intestinal permeability in IBS-D rats by targeting OCLN and ZO1. Cell Physiol Biochem 44: 2256-2268, 2017.

22 Zhang L, Feng $\mathrm{T}$ and Spicer L: The role of tight junction proteins in ovarian follicular development and ovarian cancer. Reproduction 155: R183-R198, 2018.

23 Cufi S, Vazquez-Martin A, Oliveras-Ferraros C, Martin-Castillo B, Joven $\mathrm{J}$ and Menendez JA: Metformin against TGFbetainduced epithelial-to-mesenchymal transition (EMT): from cancer stem cells to aging-associated fibrosis. Cell Cycle 9: 4461-4468, 2010.

24 Lee SH, Paek AR, Yoon K, Kim SH, Lee SY and You HJ: Tight junction protein 1 is regulated by transforming growth factorbeta and contributes to cell motility in NSCLC cells. BMB Rep 48: 115-120, 2015.

25 Martin TA, Watkins G, Mansel RE and Jiang WG: Loss of tight junction plaque molecules in breast cancer tissues is associated with a poor prognosis in patients with breast cancer. Eur $\mathrm{J}$ Cancer 40: 2717-2725, 2004.

26 Gottardi CJ, Arpin M, Fanning AS and Louvard D: The junctionassociated protein, zonula occludens-1, localizes to the nucleus before the maturation and during the remodeling of cell-cell contacts. Proc Natl Acad Sci USA 93: 10779-10784, 1996.

27 Polette M, Gilles C, Nawrocki-Raby B, Lohi J, Hunziker W, Foidart JM and Birembaut P: Membrane-type 1 matrix metalloproteinase expression is regulated by zonula occludens1 in human breast cancer cells. Cancer Res 65: 7691-7698, 2005.

28 Lesage J, Suarez-Carmona M, Neyrinck-Leglantier D, Grelet S, Blacher S, Hunziker W, Birembaut P, Noel A, Nawrocki-Raby $\mathrm{B}$, Gilles $\mathrm{C}$ and Polette $\mathrm{M}$ : Zonula occludens-1/NFkappaB/CXCL8: a new regulatory axis for tumor angiogenesis. FASEB J 31: 1678-1688, 2017.

29 Liu J, Zhang J, Li Y, Wang L, Sui B and Dai D: MiR-455-5p acts as a novel tumor suppressor in gastric cancer by downregulating RAB18. Gene 592: 308-315, 2016.

30 Ning T, Peng Z, Li S, Qu Y, Zhang H, Duan J, Wang X, Yang H, Liu R, Deng T, Bai M, Wang Y, Si Y, Zhang L, Wang X, Ge $S$, Zhou L, Ying G and Ba Y: miR-455 inhibits cell proliferation and migration via negative regulation of EGFR in human gastric cancer. Oncol Rep 38: 175-182, 2017. 
31 Wang B, Zou A, Ma L, Chen X, Wang L, Zeng X and Tan T: miR-455 inhibits breast cancer cell proliferation through targeting CDK14. Eur J Pharmacol 807: 138-143, 2017.

32 Shoshan E, Mobley AK, Braeuer RR, Kamiya T, Huang L, Vasquez ME, Salameh A, Lee HJ, Kim SJ, Ivan C, VelazquezTorres G, Nip KM, Zhu K, Brooks D, Jones SJ, Birol I, Mosqueda M, Wen YY, Eterovic AK, Sood AK, Hwu P, Gershenwald JE, Robertson AG, Calin GA, Markel G, Fidler IJ and Bar-Eli M: Reduced adenosine-to-inosine miR-455-5p editing promotes melanoma growth and metastasis. Nat Cell Biol 17: 311-321, 2015.

33 Cheng CM, Shiah SG, Huang CC, Hsiao JR and Chang JY: Upregulation of miR-455-5p by the TGF-beta-SMAD signalling axis promotes the proliferation of oral squamous cancer cells by targeting UBE2B. J Pathol 240: 38-49, 2016.
34 Wang J, Wang Y, Sun D, Bu J, Ren F, Liu B, Zhang S, Xu Z, Pang $S$ and $X u$ S: miR-455-5p promotes cell growth and invasion by targeting SOCO3 in non-small cell lung cancer. Oncotarget 8: 114956-114965, 2017.

35 Yang Q, Hou C, Huang D, Zhuang C, Jiang W, Geng Z, Wang $\mathrm{X}$ and $\mathrm{Hu} \mathrm{L}$ : miR-455-5p functions as a potential oncogene by targeting galectin-9 in colon cancer. Oncol Lett 13: 1958-1964, 2017.

36 Lewis BP, Burge CB and Bartel DP: Conserved seed pairing, often flanked by adenosines, indicates that thousands of human genes are microRNA targets. Cell 120: 15-20, 2005.

Received June 11, 2018

Revised July 3, 2018

Accepted July 9, 2018 